\title{
O ESÔFAGO DE BARRETT ASSOCIADO À ESTENOSE CÁUSTICA DO ESÔFAGO
}

\author{
Nelson Adami ANDREOLLO, Luiz Roberto LOPES, Valdir TERCIOTI Jr., \\ Nelson Ary BRANDALISE e Luiz Sérgio LEONARDI
}

RESUMO - Racional - A estenose esofágica secundária à ingestão de produtos cáusticos é freqüente no Brasil, principalmente como tentativa de suicídio. O esôfago de Barrett surge como conseqüência do refluxo gastroesofágico crônico. A literatura pesquisada mostrou que esta associação é muito rara. Casuística e Métodos - De 1981 a 2000 foram admitidos e tratados no Gastrocentro-UNICAMP (Universidade Estadual de Campinas, SP.) 120 doentes com estenose cáustica do esôfago e durante o seguimento destes, foram encontrados 9 casos associados com o esôfago de Barrett (7,5\%). O tempo de ingestão do cáustico variou de 4 a 54 anos (média de 29 anos) e eram quatro homens e cinco mulheres, oito brancos e um negro, com idade média de 57,7 anos (43 a 72 anos). Resultados - Todos os casos apresentavam disfagia e a endoscopia digestiva alta flexível mostrou áreas de estenose e seqüelas de esofagite cáustica. Três pacientes referiram sintomas de refluxo gastroesofágico, mas hérnia de hiato foi encontrada em apenas um caso. O esôfago de Barrett foi encontrado no terço médio do esôfago em três casos, acima das áreas de estenose, e nos demais, no terço distal. A disfagia foi tratada com dilatações esofágicas periódicas. Dois pacientes apresentando sintomas de refluxo grave foram submetidos a fundoplicatura à Nissen modificado através de videolaparoscopia, com bons resultados. Conclusĩes - O esôfago de Barrett nesses doentes poderia estar associado com a ingestão de cáustico, porque nem sempre esteve associado à esofagite por refluxo. É muito importante o seguimento desses doentes e realização periódica de endoscopias digestivas com biopsias do esôfago de Barrett, devido à possibilidade de malignização.

DESCRITORES - Esôfago de Barrett. Epitélio. Estenose esofágica.

\section{INTRODUÇÃO}

A ingestão acidental ou proposital de agentes corrosivos ainda é freqüente no país e significa um desafio tanto aos médicos, como à medicina preventiva em geral, tendo em vista as complicações agudas e crônicas dessa agressão ao esôfago, tais como hemorragia, broncopneumonia, perfuração, dor e estenose cicatricial. Após a fase aguda em que está presente o edema e a inflamação, segue-se a fibrose parcial ou total do órgão, acompanhada de estreitamentos, estenoses e encurtamento com danos não só na morfologia, como na sua fisiologia ${ }^{(2,8)}$.

A ingestão de hidróxido de sódio, mais comumente conhecido como soda cáustica, prevalece na literatura como uma das principais causas de estenose benigna do esôfago, atingindo duas populações: as crianças que ingerem acidentalmente o cáustico e os adultos jovens que podem estar envolvidos em tentativas de suicídio ${ }^{(6,7,19)}$.
TUCKER e YARINGTON ${ }^{(21)}$, revisando mais de 4.000 casos de ingestão de agentes corrosivos, relatam $2 \%$ de mortalidade após ingestão de soda cáustica e 20\% após ingestão de vários tipos de ácidos. POSTLETHWAIT ${ }^{(12)}$, revisando várias publicações encontrou a incidência de 5\% de estenose em 2.109 pacientes expostos a agentes corrosivos.

A evolução para a estenose do esôfago depende de alguns fatores, tais como a quantidade de cáustico ingerida, o tempo de exposição à mucosa esofagiana, as camadas do órgão que foram envolvidas e a extensão do órgão envolvido. Além disso, acredita-se que o esôfago fibrosado possa produzir hérnia de hiato por deslizamento mais freqüentemente devido à retração cicatricial, portanto, é esperado ocorrer refluxo gastroesofágico com freqüência mais elevada nesses doentes, uma vez que a hérnia é um fator entre outros envolvidos no refluxo do conteúdo cloridropéptico agressivo à mucosa esofágica ${ }^{(2,6,19)}$. Sendo assim, não é surpreendente o achado endoscópico concomitante de esofagite por refluxo nos portadores de estenose cáustica.

Disciplina de Moléstias do Aparelho Digestivo do Departamento de Cirurgia e Gastrocentro da Faculdade de Ciências Médicas da Universidade Estadual de Campinas UNICAMP, Campinas, SP.

Endereço para correspondência: Dr. Nelson Adami Andreollo - Rua Francisco Humberto Zuppi, 1234 - 13083-350 - Campinas, SP. E-mail: nandreollo@hotmail.com 
A esofagite na doença por refluxo gastroesofágico pode, por sua vez, causar outros padrões de lesão além da estenose esofágica como, por exemplo, erosões, úlceras e estenoses pépticas no esôfago e o esôfago de Barrett $(\mathrm{EP})^{(9,10,11)}$.

O EP considerado conseqüência de esofagite de refluxo crônica é uma das doenças esofágicas mais estudadas no momento, principalmente pela sua relação com o desenvolvimento de adenocarcinoma. Endoscopicamente é caracterizado por epitélio de cor avermelhada na extensão de no mínimo 2 a $3 \mathrm{~cm}$ do esôfago distal, que se destaca do epitélio esofágico normal ${ }^{(11,13,20)}$.

Enfim, nos portadores de estenose cáustica do esôfago presumivelmente somam-se fatores predisponentes para o desenvolvimento do EP. Revendo a literatura, porém, foi encontrada uma única publicação completa a respeito, de autoria de SPECHLER et al. ${ }^{(18)}$, em 1981, descrevendo um caso com EP no terço médio do esôfago concomitante com a história pregressa de ingestão de cáustico e conseqüente estenose cáustica.

O objetivo do presente trabalho foi analisar a ocorrência do EP entre os portadores de estenose cáustica do esôfago no GastrocentroUNICAMP - Universidade Estadual de Campinas, SP.

\section{CASUÍSTICA E MÉTODOS}

No período de 1981 a 2000 foram admitidos e tratados no Gastrocentro - UNICAMP 120 doentes apresentando estenose cáustica do esôfago de várias etiologias, todos seguidos e submetidos regularmente a dilatações esofágicas com auxílio de endoscopia digestiva $^{(2)}$. Entre eles, foram identificados em nove casos a ocorrência do EP $(7,5 \%)$. A confirmação foi através de biopsias adequadamente colhidas das áreas suspeitas e estudos histopatológicos.

A faixa etária dos doentes variou de 43 a 72 anos (média de 57,7 anos), que se distribuíram quanto ao sexo em quatro homens e cinco mulheres, e quanto à raça, em oito brancos e um negro. $\mathrm{O}$ tempo de ingestão neste grupo variou de 4 a 54 anos (média de 29 anos) e a etiologia para a estenose foi, em todos os casos, a ingestão de soda cáustica.

\section{RESULTADOS}

Todos os casos apresentavam disfagia de variável intensidade e a endoscopia digestiva alta flexível mostrou áreas de estenose e seqüelas de esofagite cáustica. Três doentes referiram sintomas de refluxo gastroesofágico, mas hérnia de hiato foi verificada em apenas um caso. A extensão endoscópica do EP variou de 1,5 a $3,5 \mathrm{~cm}$.

O tratamento para alívio da disfagia consistiu de dilatações esofágicas periódicas, mediante programa de dilatações instituído para cada doente, conforme a intensidade dos sintomas e a gravidade das estenoses. Dois casos apresentando esofagite grau C (classificação de Los Angeles) e sintomas de refluxo grave foram submetidos a tratamento cirúrgico, tendo sido indicada a fundoplicatura à Nissen modificada, através de videolaparoscopia ${ }^{(9)}$, com melhora significativa da sintomatologia.

Quanto ao local de ocorrência do EP no esôfago, a maioria deles apresentava a lesão no terço inferior, acompanhando o local da estenose. Em três casos, porém, o EP situava-se no terço médio do órgão, distante portanto do local da estenose.

Todos os doentes continuam em seguimento ambulatorial, sendo periodicamente submetidos a novas biopsias na tentativa de identificar displasias ou malignização.

\section{DISCUSSÃO}

Vários autores têm tentado realizar nos últimos anos estudos epidemiológicos para avaliar a prevalência do EP tanto na população em geral, como em séries clínicas de doentes portadores de esofagite de refluxo ou outros sintomas gastrointestinais ${ }^{(1,10,11,13,17)}$

Revisão realizada no Gastrocentro-UNICAMP mostrou prevalência de 22,4 casos por $100.000 /$ ano $^{(1)}$. A revisão da literatura mostra que entre $23 \%$ e $40 \%$ dos portadores de EP não têm quaisquer sintomas dispépticos e que esses casos não são identificados, até que a endoscopia digestiva seja realizada por outras razões. Além disso, é referido que entre $10 \%$ e $20 \%$ dos casos, o diagnóstico é feito devido a alguma complicação, como úlcera, estenose e neoplasia associada ${ }^{(3,10,11)}$

O EP associado ao refluxo gastroesofágico crônico tem possibilidade de sofrer malignização ao longo dos anos, em percentagens variáveis referidas na literatura, se não for adequadamente tratado ${ }^{(3,10,14)}$

Por outro lado, as estenoses esofágicas de origem cáustica são freqüentes em todo o território nacional, ocorrendo em $5 \%$ a $33 \%$ dos casos registrados. O mais comum é a própria soda cáustica para uso doméstico, porém, outros produtos, tais como amoníaco, acído nítrico, ácido muriático, ácido clorídrico, soluções para baterias e outros produtos de limpeza contendo substâncias cáusticas também são ingeridos. A evolução para estenose geralmente ocorre nas primeiras 2-8 semanas, freqüentemente é de grande extensão e persiste por longo tempo ${ }^{(2,6,19)}$

O tratamento inicial é a tentativa de dilatações e caso não ocorra melhora da disfagia, com queda do estado geral e emagrecimento, o tratamento cirúrgico deverá ser logo indicado. O progresso e os avanços trazidos pelos novos dilatadores e métodos de dilatações associados à endoscopia flexível foram notáveis nos últimos anos, de modo que apenas 1 entre aproximadamente 20 doentes que ingerem caústicos têm necessidade de tratamento cirúrgico para reconstrução do trânsito digestivo e retorno à alimentação por via oral ${ }^{(2,7)}$. 
É importante lembrar que o doente portador de estenose cáustica deverá ter acompanhamento médico e endoscópico por longos anos, não somente para controle da disfagia, mas porque a literatura descreve nesses casos a ocorrência mais freqüente de carcinoma epidermóide, principalmente após 30 a 40 anos do incidente, sendo que essa associação, segundo algumas publicações, não ultrapassa $3 \%$ a $5 \%(2,6,19)$.

SPECHLER et al. ${ }^{(18)}$ descreveram o caso de doente masculino de 67 anos que havia ingerido soda cáustica há cerca de 40 anos. Realizaram seguimento endoscópico durante vários anos, e aproximadamente 20 anos após a ingestão passou a apresentar área bem definida de epitélio atípico, bem diferente do epitélio esofágico e no terço médio do esôfago, cujas biopsias mostraram tratar-se de EP. $\mathrm{O}$ doente em questão não apresentava hérnia hiatal e nem esofagite de refluxo. A hipótese desses autores para o surgimento do EP foi de que se tratava de mucosa gástrica ectópica secundária à ingestão da soda cáustica. A mucosa esofágica danificada pelo cáustico teria sido reepitelizada às custas de proliferação de restos heterotrópicos de mucosa gástrica no terço médio do esôfago.

FISHER et. al. ${ }^{(5)}$, em 1983, descreveram a indicação de cintilografia com tecnécio-99 para determinar a presença e extensão de EP nos dois terços distais do esôfago em um doente que tinha história prévia de ingestão de soda cáustica.

Assim, na presente casuística o EP em seis dos nove casos estudados pode estar associado diretamente com a ingestão de cáustico, porque não apresentavam concomitante esofagite de refluxo.
Além disso, em três desses seis casos essa metaplasia não estava situada no terço distal do esôfago, como é habitualmente encontrada na esofagite por refluxo.

Este estudo mostra que o tempo para o aparecimento de transformações na mucosa características do EP é bem longo, cerca de 30 anos após o episódio agudo da ingestão do cáustico.

No caso de malignização desse EP associado à ingestão cáustica, certamente o tipo histopatológico será de adenocarcinoma, diferindo, portanto, do que habitualmente tem sido encontrado, que é o carcinoma epidermóide. Encontrou-se na literatura um único trabalho que relata a ocorrência de adenocarcinoma no terço médio do esôfago de doente com história prévia de ingestão de cáustico, feito por CORNET et al. ${ }^{(4)}$, em 1976, que não referem a ocorrência do EP associado, mas estranham muito este fato, enfatizando que a malignização seria em decorrência do produto ingerido.

No sentido de prevenir a degeneração maligna do EP ao longo dos anos, alguns autores recomendam a sua ablação endoscópica com laser, eletrocauterização ou terapia fotodinâmica ${ }^{(15,16)}$. Entretanto, especificamente no caso de EP associado à ingestão de cáusticos não há relatos na literatura.

Concluindo, mais uma vez a soda cáustica desponta como agente agressor do esôfago, o que reforça os trabalhos para a educação e prevenção desses acidentes, principalmente entre as crianças. Além disso, é muito importante que esses doentes realizem endoscopias digestivas periódicas, mesmo se estiverem assintomáticos, para diagnóstico de ocorrência de EP associado ou eventual malignização.

Andreollo NA, Lopes LR, Tercioti Jr V, Brandalise NA, Leonardi LS. Barrett's esophagus associated to caustic stenosis of the esophagus. Arq Gastroenterol 2003;40(3): 148-151

ABSTRACT - Background - The esophageal stenosis secondary to the ingestion of caustic products is frequent in Brazil, mainly due to an attempt suicide The Barrett's esophagus is consequence of the chronic gastroesophageal reflux. The literature consulted showed that are a rare association. Casuistic and Methods - From 1981 to 2000 were admitted and treated in the "Gastrocentro" State University of Campinas, SP, Brazil, 120 patients presenting caustic stenosis of the esophagus, and during the follow-up were found nine cases with Barrett's esophagus associated (7,5\%). The time of caustic ingestion varied from 4 years to 54 years (mean 29 years), and they were four males and five females, eight whites and one black, with the mean age of 57,7 years ( 43 to 72 years). Results - All the cases presented dysphagia and the flexible upper endoscopy showed stenotic areas and caustic esophagitis sequels. Three patients referred symptoms of gastroesophageal reflux, but hiatus hernia were found in only one case. The Barrett's esophagus was found in the middle third of the esophagus above the stenotic areas in three cases, and in the distal third in six cases. The dysphagia was treated with periodic esophageal dilatations. Two patients presenting severe symptoms of reflux were submitted to modified Nissen fundoplication by videolaparoscopy, with good results. Conclusions - The Barrett's esophagus in these patients could be associated to the caustic ingestion, because it is not situated in the distal third of the esophagus, as commonly found in reflux esophagitis. Its is very important the follow-up and biopsies of the Barrett's esophagus, due to the possibility of malignancy.

HEADING - Barrett esophagus. Epithelium. Esophageal stenosis. 


\section{REFERÊNCIAS BIBLIOGRÁFICAS}

1. Andreollo NA, Miquelino M, Lopes LR, Trevisan MAS, Brandalise NA, Leonard LS. Incidência e epidemiologia do epitélio de Barrett no Gastrocentro/UNICAMP. Arq Gastroenterol 1997;34:22-6.

2. Andreollo NA, Lopes LR, Nogutti R, Brandalise NA, Leonardi LS. Tratamento conservador das estenoses benignas do esôfago através de dilatações. Análise de 500 casos. Rev Assoc Med Bras 2001;47:236-43.

3. Bremner CG, Bremner RM. Malignant degeneration of Barrett's esophagus: current concepts on the pathogenesis of Barrett's esophagus. Dis Esophagus 1995;8:79-85.

4. Cornet A, Barbier J, Grenier G, Elhadad A, Breaute H, Carnot F. Caustic burn and adenocarcinoma of the esophagus. Sem Hop 1976;52:1999-2003.

5. Fisher DR, Preston DF, Robinson RG, Ginsberg BW. Barrett's esophagus complicating by ingestion. Demonstration by pertechnetate scintigraphy. Clin Nucl Med 1983;8:550-2.

6. Gumaste VV, Dave PB. Ingestion of corrosive substances by adults. Am J Gastroenterol 1992;87:1-5.

7. Ishioka S. Estenoses benignas do esôfago. In: Sociedade Brasileira de Endoscopia Digestiva - SOBED, editor. Endoscopia digestiva. Rio de Janeiro: Medsi; 1994 p. 85-99.

8. Kikendall JW. Caustic ingestion injuries. Gastroenterol Clin North Am 1991;20:847-57.

9. Lopes LR, Brandalise NA, Andreollo NA, Leonardi LS. Tratamento cirúrgico videolaparoscópico da doença do refluxo gastroesofagiano: técnica de Nissen modificada - Resultados clínicos e funcionais. Rev Assoc Med Bras $2001 ; 47: 141-8$
10. Pera M, Duranceau A. Malignant degeneration of Barrett's esophagus: epidemiology of Barrett's esophagus and esophageal adenocarcinoma. Dis Esophagus 1995;8:86-92.

11. Phillips RW, Wong RKH. Barrett's esophagus. Natural history, incidence, etiology and complications. Gastroenterol Clin North Am 1991;20:791-815.

12. Postlethwait RW. Chemical burns of the esophagus. In: Postlethwait RW. Surgery of the esophagus. New York: Appleton Century Crofts; 1979. p. 287.

13. Provenzale D, Kemp JA, Arora S, Wong JB. A guide for surveillance of patient with Barrett's esophagus. Am J Gastroenterol 1994;89:670-80.

14. Reid B. Barrett's esophagus and esophageal adenocarcinoma. Gastroenterol Clin North Am 1991;20:817-33

15. Sampliner RE, Jaffe P. Malignant degeneration of Barrett's esophagus: the role of laser ablation and photodynamic therapy. Dis Esophagus 1995;8:104-8.

16. Salo JA. Malignant degeneration of Barrett's esophagus: the role of laser ablation and anti-reflux surgery. Dis Esophagus 1995;8:109-10.

17. Spechler SJ, Sperber H, Doos W, Schimmel EM. The prevalence of Barrett's esophagus in patients with chronic peptic esophageal strictures. Dig Dis Sci 1983;28:769-74.

18. Spechler SJ, Schimmel EM, Dalton JW, Doos W, Trier JS. Barrett's epithelium complicating lye ingestion with sparing of the distal esophagus. Gastroenterology 1981;81:580-3.

19. Talbert JT. Estenoses cáusticas do esôfago. In: Sabiston DC Jr, Lyerly HK editores. Tratado de cirurgia. 15 ed. Rio de Janeiro: Guanabara Koogan; 1999. p. 732-7.

20. Tolentino MM, Faifer JG, Trentini EA. Doença do refluxo gastroesofágico. In Dani R, editor. Gastroenterologia essencial. Rio de Janeiro: Guanabara Koogan; 1998. p. 62-74.

21. Tucker JA, Yarington CT. The treatment of caustic ingestion. Otolaryngol Clin North Am 1979:12:343-50.

Recebido em 12/3/2002 Aprovado em 23/1/2003 